\title{
A Data-Driven Framework to Predict Fused Filament Fabrication Part Properties Using Surrogate Models and Multi-Objective Optimisation
}

\section{Yongjie Zhang}

Nanyang Technological University

Joon Phil Choi

Nanyang Technological University

Seung Ki Moon ( $\nabla$ skmoon@ntu.edu.sg )

Nanyang Technological University https://orcid.org/0000-0002-2249-7500

\section{Research Article}

Keywords: Additive manufacturing, Fused Filament Fabrication, Mechanical properties, Multi-objective optimisation, Surrogate models

Posted Date: December 20th, 2021

DOI: https://doi.org/10.21203/rs.3.rs-1159839/v1

License: (a) (1) This work is licensed under a Creative Commons Attribution 4.0 International License. Read Full License 


\title{
A data-driven framework to predict fused filament fabrication part properties using surrogate models and multi-objective optimisation
}

\author{
Yongjie Zhanga, Joon Phil Choi ${ }^{\mathrm{b}}$, Seung Ki Moon ${ }^{\mathrm{a}, *}$ \\ ${ }^{a}$ Singapore Centre for 3D Printing, School of Mechanical and Aerospace Engineering, Nanyang \\ Technological University, Singapore \\ ${ }^{b}$ Department of 3D Printing, Korea Institute of Machinery \& Materials, Daejeon 34103, Republic of \\ Korea
}

*Corresponding author / E-mail: skmoon@ntu.edu.sg ORCID: 0000-0002-2249-7500

\begin{abstract}
In additive manufacturing (AM), due to large number of process parameters and multiple responses of interest, it is hard for AM designers to attain optimal part performance without a systematic approach. In this research, a data-driven framework is proposed to achieve the desired AM part performance and quality by predicting part properties and optimizing AM process parameters effectively and efficiently. The proposed framework encompasses efficient sampling of design space and establishing the initial experiment points. Based on established empirical data, surrogate models, are used to characterise influence of critical process parameters on responses on interest. Further, process maps can be generated for enhancing understanding on the influence of process parameters on responses of interests and AM process characteristics. Subsequently, multi-objective optimisation coupled with a multi criteria decision making technique is applied to determine an optimal design point, which maximises the identified responses of interest to meet the part functional requirements. A case study is used to validate the proposed framework for optimising an ULTEM $^{\text {TM }} 9085$ fused filament fabrication part to meet its functional requirements of surface roughness and mechanical strength. From the case study, results indicate that the proposed approach is able to achieve good predictive results for responses of interest with a relatively small dataset. Further, process maps generated from the surrogate model provide a visual representation of the influence between responses of interest and critical process parameters for FFF process, which traditionally requires multiple investigations to arrive at similar conclusions.
\end{abstract}

Keywords: Additive manufacturing, Fused Filament Fabrication, Mechanical properties, Multi-objective optimisation, Surrogate models 


\section{List of nomenclatures}

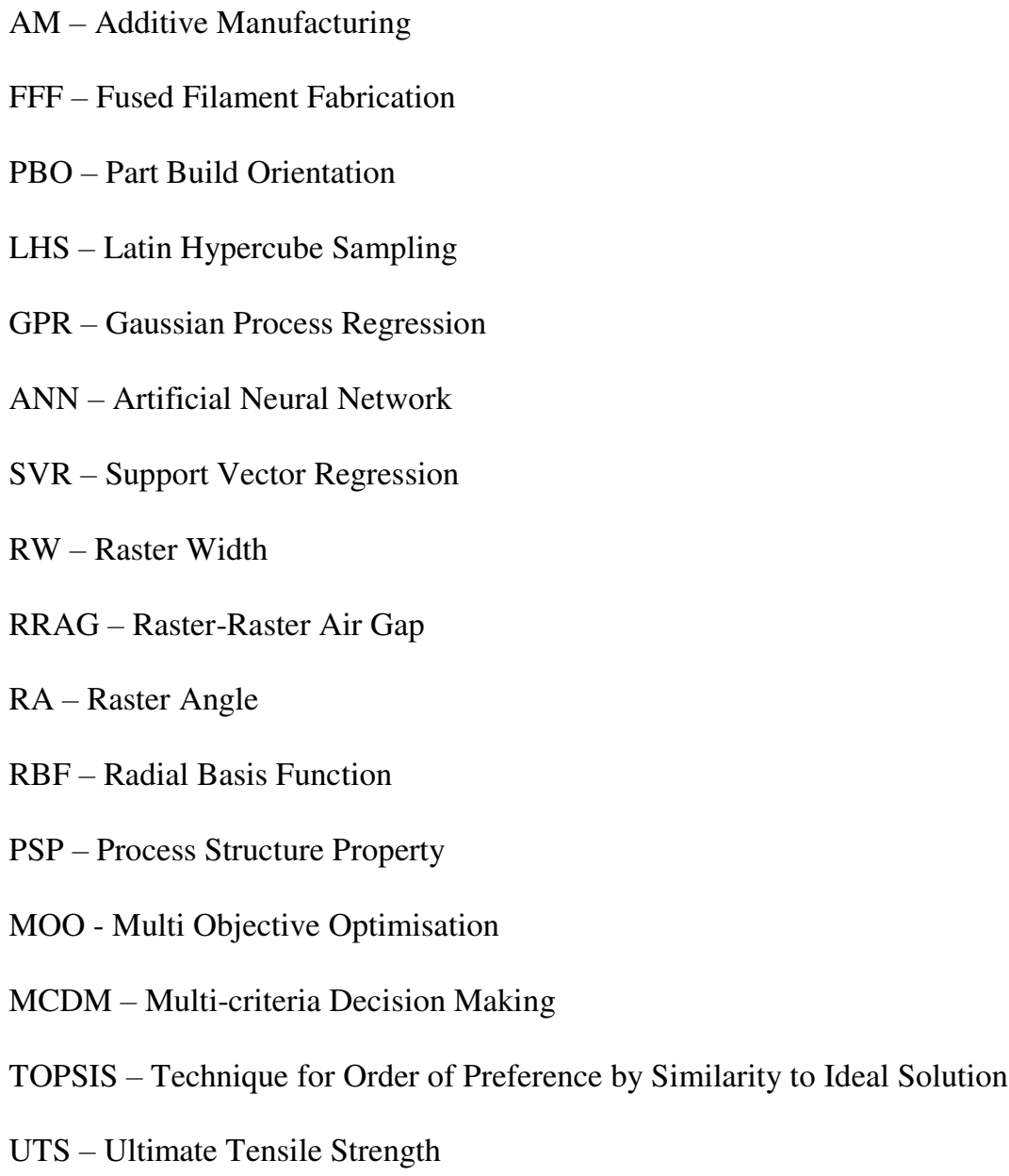

\section{Introduction}

Additive manufacturing (AM) is a layered manufacturing technique where digital files are converted into physical parts [1,2]. Traditionally, AM has been used as a rapid prototyping technique which reduces developmental cycle time for product design. In recent years, popularity of AM has risen exponentially as the technology matures, which resulted in higher adoption for printing direct end use parts. Complex structure and topology which are near impossible to be manufactured using conventional manufacturing techniques can now be produced in a cost effective and efficient manner using AM technologies [3-8].

To achieve desired part functional requirements, AM designers typically have to perform tradeoff analysis between responses of interest, (e.g. surface roughness requirements, mechanical strength, material usage, build time, etc.) by varying process parameters and part build orientations (PBO) [9-11]. In order to achieve the optimised AM part performance, AM designers will need to have a good understanding of design space, and the impact of varying process parameters on the final part properties. However, an 
optimisation task is complicated by the sheer number of process parameters that can be changed and varied. Further, due to the strong presence of Process Structure Property (PSP) linkage, both embodiment design and process parameters play a critical role in influencing the mechanical properties of a final part [12]. However, the AM designers often rely on their experiences or trial and error for selecting process parameters [13]. Confounding between the parameters and potential non-linearity relationships between parameters and response of interest may not be fully understood and characterised through simple trial and error experiments [14]. Thus, to obtain optimal AM part performance, the following critical steps are required. Firstly, a structured approach is necessary to systematically characterise the design space efficiently and effectively. Secondly, models should be built to estimate and predict AM part properties during the design phase. Lastly, optimisation of AM parts design and process parameters should be based on empirical evidence and data. A knowledge management framework or methodology can then be used to capture the on-going data collection efforts and further improve on the understanding and characterisation of the design space.

To aid the AM designers in designing optimised parts, data driven methodologies have been proposed. These data driven methodologies combine and aggregate data points, and analyse them using algorithms to derive meaningful and actionable insights, which can aid in characterisation and optimisation efforts to improve AM part performance [15-19]. Further, data driven methodologies are an effective and efficient way of exploration and exploiting the design space [20, 21]. One of the key methodologies is surrogate modelling, which utilises techniques such as Gaussian Process Regression (GPR) and Support Vector Regression to model the system response. Through the methodologies, cheap and fast prediction and exploration even within high dimensional design spaces are made possible. Typical run time for physics model-based approach ranges from hours to days, whereas typical run time for surrogate models is a magnitude faster, ranging from seconds to minutes [22, 23]. As a result, surrogate models are commonly utilised for prediction and optimisation, and current state of the art literatures are discussed in the following paragraphs.

Rankouhi, Jahani [14] applied surrogate modelling to predict the optimal process parameters for manufacturing compositional gradient for $316 \mathrm{~L}-\mathrm{Cu}$ multi material part. GPR with a convoluted covariance function was trained for the prediction, and it was found that non-linear relationship exists between process parameters and response. Yang, Barrett [24] developed a model to predict solidification morphology, which aimed to aid AM designers tailor and optimise microstructure to achieve a range of mechanical properties 
for different applications. Jiang, Hu [25] used Artificial Neural Network (ANN) to predict the printable bridging length for a given set of parameters.

Pandey, Thrimurthulu [11] utilized a non-dominated sorting genetic algorithm (NSGA-II) to optimize the PBO with an objective to reduce the surface roughness and minimizing the build time. The surface roughness was estimated using surrogate modelling of empirical data. Similarly, Khodaygan and Golmohammadi [9] applied NSGA-II to search for the optimal PBO with build time and surface roughness as the objective function. Jothibabu and Kumar [26] evaluated the effect of process parameters on the tensile strength using 3 different surrogate models (namely Kriging, Radial Basis Function, Polynomial Responses Surface) and compared the prediction accuracy among the models. Sobol sensitivity analysis was then carried out to determine the parameter that had the greatest impact on the part strength. Vahabli and Rahmati [27] applied Radial Basis Function Neural Network (RBFNN) to predict the surface quality of fused filament fabrication (FFF) parts by varying the part build orientation. Liu and Wang [28] combined a model-based approach and surrogate modelling to construct a stochastic multi-level modelling framework for predicting the performance of a FFF part. A modified Classical Laminate Theory (CLT) was combined with Kriging surrogate modelling was utilized.

However, many literatures often fail to elaborate on a general framework which can be applied to multifarious responses of interest, an AM designer may wish to consider. Many literatures, including the ones discussed above, lacked generality and only tune and demonstrate the applicability of their methodology on a niche response(s) of interest. Further, copious data points are required to ensure models can be sufficiency accurate in the enlarged design space. Many of these literatures fail to touch on the data collection and aggregation methodologies and strategies.

Among the AM technologies, Fused Filament Fabrication, which is classified under material extrusion under the 7 process categories as defined in ISO/ASTM 52900, is one of the most popular and most utilised primarily due to ease of operation and low operating cost of a FFF printer [29, 30]. In the recent years, FFF has been increasingly adopted for printing direct end use parts, and has been actively been used to produce structures such as unmanned aerial vehicles and even producing critical parts such as turbine vanes [31-37]. However, there is a lack of comprehensive framework for designing a FFF part, as identified above and in authors' previous work Zhang and Moon [12]. Key elements, such as process maps and knowledge management components, are not investigated and researched in many FFF literatures. The process maps will enable AM designs to gain valuable insights and assist them to understand the process 
characteristics [38-41]. Unfortunately, similar insightful process maps are not well defined in FFF. In addition, the prediction and optimisation of strength of FFF parts are performed in isolation with FFF part surface roughness. Typically, these 2 responses are of great interest to the AM designers as they design end use parts, as the responses determine the mechanical performance and the general aesthetics of the AM part. Further, many literatures discussing part surface roughness focuses mainly on PBO, without given other process parameters due considerations [9, 11, 27].

In this research, a data-driven framework is proposed to achieve the desired AM part performance and quality by predicting part properties and optimizing AM process parameters. In the proposed framework, a systematic approach is utilised to characterise design space efficiently and effectively, upon which surrogate models are built to estimate and predict responses of interest during AM part design phase. A knowledge management methodology is also incorporated within the proposed framework, which enables continuous improvement in the predictive accuracy of the established surrogate model. To validate the proposed framework and address the aforementioned research gaps identified in FFF, a case study is performed to identify the trade-off between mechanical strength and surface roughness in FFF parts.

The remaining of the paper is structured as follows. The proposed data driven framework is introduced in Section 2. Section 3 describes the experiment setup and validation of the proposed framework and discusses the results. The paper concludes with a summary of the key findings and contributions and discusses areas for future work in Section 4.

\section{A data-driven framework}

As shown in Fig.1, the proposed data-driven framework 6 key steps. To build a surrogate model, a database is generated by aggregating empirical data in Step 1. An efficient sampling strategy is selected to search the design space efficiently. Alternatively, existing data points can be incluNext, in Step 2, surrogate models are created from the sampled data points. Each surrogate model (SM) is created for each response of interest. Thus, $N$ surrogate models are established for $N$ responses of interest. In Step 3, the surrogate model is validated to determine its accuracy, and if necessary, further updates to the surrogate model can be performed to enhance its predictive accuracy. Process maps are generated to enhance the understanding of the process characteristics.

Once the surrogate models are validated, the models can be used for multi-objective optimisation. The part functional requirements are identified and translated into response of interests (e.g., mechanical strength, surface roughness, etc.), In Step 4, these requirements are translated into responses of interest and 
their corresponding fitness function is selected. In Step 5, multi-objective optimisation is conducted to maximise the overall fitness function, and the resultant pareto front from the optimisation run is ranked and evaluated, and then the optimal design point is chosen and implemented. In Step 6, the optimised part is manufactured and the performance can be validated against the process maps generated. Further, the data point can be incorporated back into the empirical database for improving the accuracy of the model.

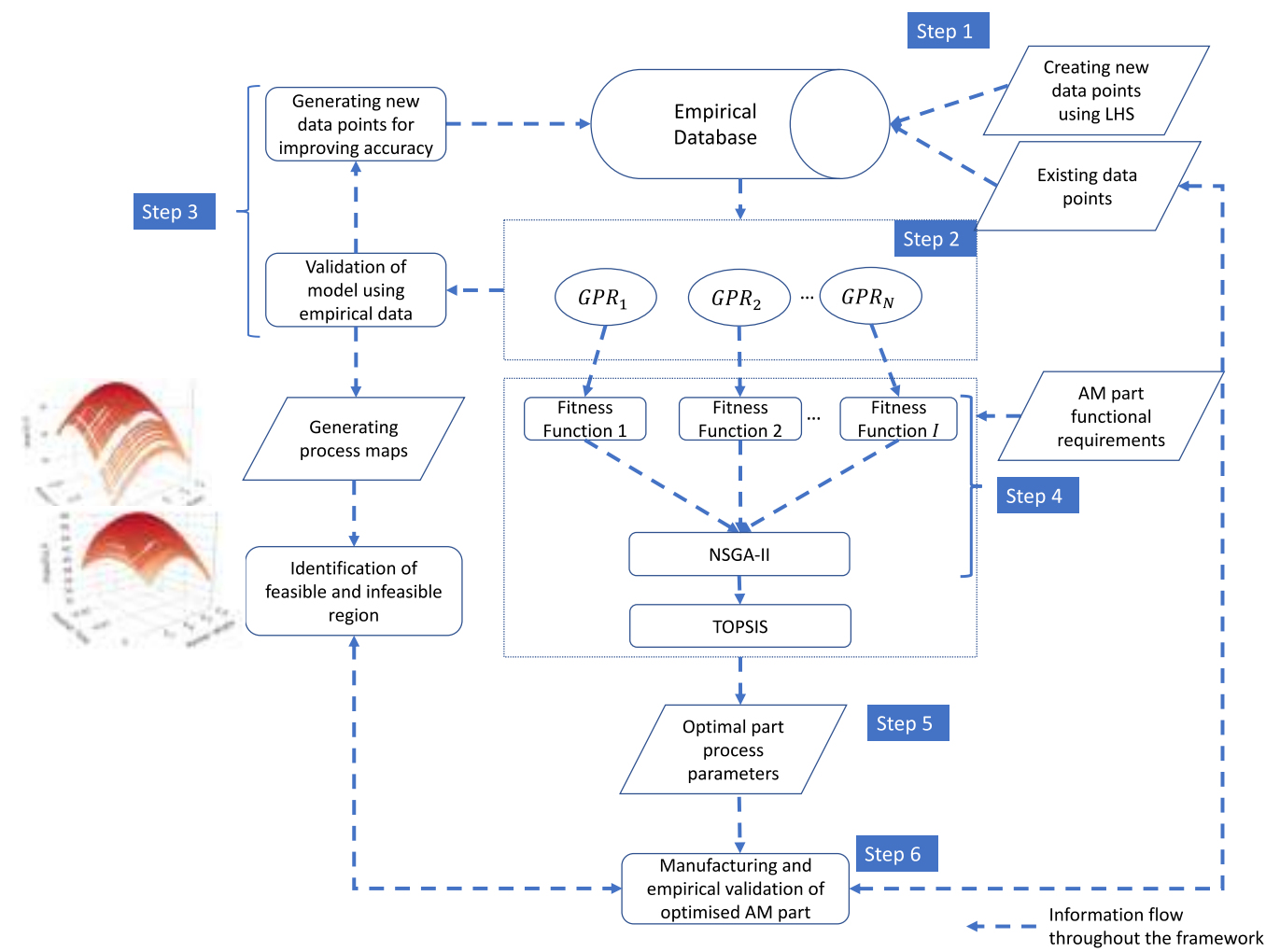

Fig. 1 Framework for data driven design of AM part

\subsection{Building empirical database}

Latin Hypercube Sampling (LHS) is commonly used to realise space filling design for surrogate models to ensure that design space is sufficiently sampled and to allow the model to be able to provide good predictive accuracy. A Latin Hypercube can be implemented by dividing the design space into $n$ equally probable intervals, and through drawing $n$ sample points, and this process is repeated for $k$ times, where $k$ is the number of variables. In contrast with traditional methods such as Design of Experiment methodology, as number of factors increase, the number of runs or data points increases exponentially. Although there are strategies to reduce the number of runs, such as fractional factorial, these may result in reduced accuracy $[42,43]$. 


\subsection{Surrogate modelling}

Surrogate modelling is a popular technique for characterization of design space. Empirical data gathered from computationally and time-consuming multi-physics simulation or field observations are used to build surrogate models for prediction and optimisation. The surrogate models are significantly simpler than simulation models and actual process but yet, possesses sufficient fidelity to predict the response [13, $20,38,44,45]$. In the surrogate modelling, physics-based simulation or field observation can be represented by $f(\boldsymbol{x})$ within a design space $\mathfrak{R}$, and the process outcome $\boldsymbol{y}$ for some input $\boldsymbol{x}$. A surrogate model approximates the input output relation using $\bar{f}(\overline{\boldsymbol{x}})$. Often, there is an error, $\varepsilon$, a deviation from results $\boldsymbol{y}$ for inputs $\boldsymbol{x}$, due to inaccuracies and limitations of the surrogate model.

Many surrogate modelling techniques build upon Bayesian inference. Bayesian inference methods revolves around updating prior knowledge about distribution of a function $Y$ based on observations $D$ and the resultant posterior density is as per $p(Y \mid D)$. Prior density, $p(Y)$, can be derived based on prior knowledge of the process [46]. There are several types of Bayesian methods that are available, such as Bayesian Network, Bayesian Calibration, and Gaussian Process Regression (GPR) [47]. GPR is a nonparametric nonlinear modelling method and has been commonly used as a surrogate modelling tool for computer experiments. And GPR has been gaining popularity in machine learning due to its superiority for out of sample test performance, and uncertainty characterization and management [14, 48, 49]. Further, GPR provides predictive variance which allows modelling of predictive uncertainty which can be used for further optimization and decision making [13, 43]. The following section discuss in detail the implementation of GPR in the proposed framework.

\subsubsection{Gaussian Process Regression}

A simple schematic of GPR is as shown in Fig. 2. GPR assumes the output of function $Y$ at input $x$ can be expressed in the form of $Y=f(x)+\varepsilon$, where $\varepsilon$ is a normally distributed independent and identically distributed error term. GPR utilizes the Bayesian inference with a prior knowledge $p(Y)$ on a space of functions and assigns a probability onto the set of functions [50]. Bayes' theorem is used to infer the posterior distribution $p(Y \mid D)$ for an empirical dataset, for a given kernel or covariance distribution between datapoints $p(D)$.

Equation (1) defines the Gaussian Process prior, $Y$,

$$
Y \sim \mathcal{N}_{n}\left(\mu(x), \Sigma_{n}\right)
$$


Where $\mu(x)$ is the mean and $\Sigma_{n}$ is the covariance matrix, which is a $n \times n$ positive definite matrix. The prior mean, $\mu(x)$, can take the form of 0 mean, a constant or a regression model. However, selecting the appropriate covariance distribution as $\Sigma_{n}$ is important, as has been shown to achieve good performance even if the prior mean function is varied $[13,51]$. Once the training data $D_{N}$ has been factored in, the posterior distribution becomes:

$$
Y(\mathcal{X}) \mid D_{N} \sim \mathcal{N}_{n^{\prime}}(\mu(\mathcal{X}), \Sigma(\mathcal{X}))
$$

Where the mean and covariance structure are as shown in Equations (3) and (4) respectively:

$$
\begin{gathered}
\mu(\mathcal{X})=K_{\theta}\left(\mathcal{X}, \mathcal{X}_{n}\right) K_{\theta}{ }^{-1} Y_{n} \\
\Sigma(\mathcal{X})=\hat{\tau}^{2}\left[K_{\theta}(\mathcal{X}, \mathcal{X})-K_{\theta}\left(\mathcal{X}, \mathcal{X}_{n}\right) K_{\theta}{ }^{-1} K_{\theta}\left(\mathcal{X}, \mathcal{X}_{n}\right)^{\top}\right]
\end{gathered}
$$

The surrogate model is then trained with the database and its performance can be validated against new empirical data. The process can be repeated to establish the surrogate model for different responses.

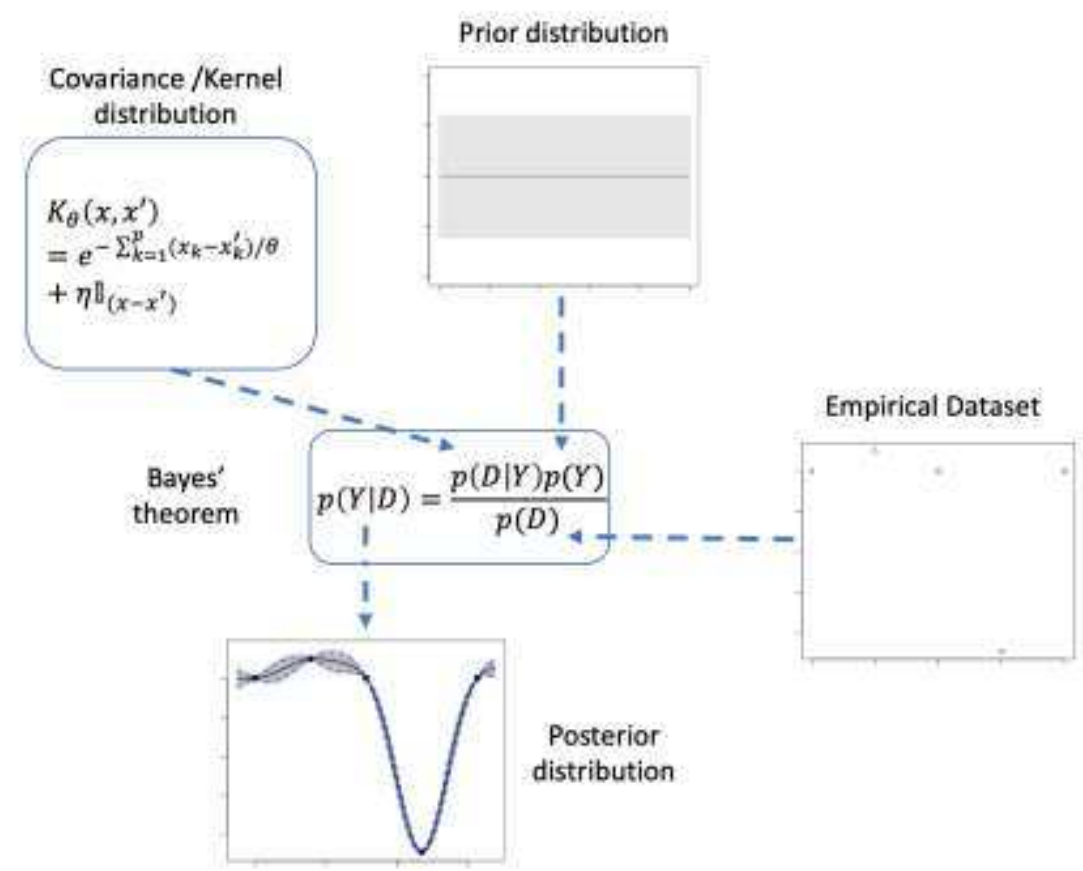

Fig. 2 Schematic for Gaussian Process Regression

\subsection{Updating of database}

When the surrogate models are first established, validation is required to ensure that the models are properly trained, and their performance is up to expectation. Validation techniques include k-fold cross validation, leave-one-out cross validation, etc. [52-55]. 
Once surrogate models are built for the response of interest, the models can be updated to further reduce potential bias and enhance their predictive accuracy. The process of updating the database and the surrogate models can be completed either through generating new empirical data at design points or based off on data points in existing database and literature.

The surrogate models can be used to analyse and determine additional design points required to minimise the overall predictive errors of the model, through maximisation of the expected improvement (EI). Equation (5) shows the closed form solution for EI when $y_{n} \mid D_{n}$ is approximately a gaussian distribution, which corresponds to the derived GPR surrogate model $[48,56]$.

$$
E I(x)=\left(f_{\min }^{n}-\mu_{n}(x)\right) \Phi\left(\frac{f_{\min }^{n}-\mu_{n}(x)}{\sigma_{n}(x)}\right)+\sigma_{n}(x) \phi\left(\frac{f_{\min }^{n}-\mu_{n}(x)}{\sigma_{n}(x)}\right)
$$

Where $\phi$ is standard gaussian probability density function and $\Phi$ is gaussian cumulative density function. In implementation, predictive mean $\mu_{n}(x)$ and uncertainty $\sigma_{n}(x)$ are factored into the calculation and the EI is highest when the $\mu_{n}(x)$ is below $f_{\min }^{n}$ or when $\sigma_{n}(x)$ is high. Multi search can be utilised to search for locations where EI is the highest, and additional experimentation data points can be performed at the locations to decrease the associated uncertainty and improve the predictive accuracy of the surrogate models.

On the other hand, literature and existing experimentation data points can be incorporated to the surrogate models to further enhance their predictive accuracy. Methods such as fast decomposition of covariance matrix $K_{\theta}$ enables established surrogate models to be updated quickly, even when the covariance matrix is large [48]. However, these existing data will need to be selected, processed and reviewed based on domain knowledge of the process and expert judgement to ensure the fidelity [45].

Once established, surrogate models can be utilised to generate predictive process maps. As discussed in aforementioned section, process maps are commonly used in AM processes to understand the characteristics of the process and identify the desirable range of parameters and the optimal operating regions. Further, the process maps can also be used identify undesirable operating regions where defects and issues with poor print quality commonly occurs. This allows AM designers to have a better grasp of the relationship between the process parameters, their significance and impact of change and search the design space for optimal design points [57]. From the process maps, the influence of parameters on response of interest and confounding between parameters can be determined, which can also be used for robust parameter design. 


\subsection{Multi-objective Optimisation}

Functional requirements of an AM part frequently encompass mechanical properties, aesthetics, and build time. To optimise these various responses of interests simultaneously, multi-objective optimisation (MOO) can be used and formulated as such,

$$
\begin{gathered}
\text { Minimise } Y(\boldsymbol{x})=\left\{y_{1}(\boldsymbol{x}), y_{2}(\boldsymbol{x}), y_{m}(\boldsymbol{x}) \ldots\right\}^{T} \\
\text { s.t. } f_{j}(\boldsymbol{x})=0, j=1, \ldots, n \\
g_{i}(\boldsymbol{x}) \leq 0, i=1, \ldots, l \\
\boldsymbol{x} \in \Omega
\end{gathered}
$$

Where $\boldsymbol{x}$ is a decision vector, $Y(\boldsymbol{x})$ consists of $m$ objective functions, $f_{j}(\boldsymbol{x})$ is the $j^{\text {th }}$ equality constraint, $g_{i}(\boldsymbol{x})$ is the $i^{\text {th }}$ inequality constraint and $\Omega$ is decision space.

In MOO, improvement of one objective function may lead to deterioration of another [58]. In FFF, conflicting objectives can arise between responses of interest, such as between surface roughness of part, build time and mechanical strength. The build time is correlated with the part build height, and good surface finishes on a plane is often achieved when the plane is orientated parallel to the $\mathrm{Z}$ axis. The mechanical strength, however, will suffer as a result. To ensure an optimal trade-off, a multi-objective genetic algorithm is used to conduct the optimisation, due to its performance as compared to other optimisation algorithms. Advantages include not requiring any derivative information and hence reducing the computational burden, and relative ease to implement [59]. Examples of such genetic algorithms include Vector evaluated genetic algorithm, a tournament selection technique, etc. [60].

In the proposed framework, a non-dominated sorting genetic algorithm (NSGA-II) introduced by Deb, Pratap [61] is used to obtain a Pareto solution for the conflicting MOO. NSGA-II has been widely used in literature for multi objective optimisation and the efficiency and effectiveness validated $[9,11,62$, 63]. Further, the algorithm enables maintaining diversity of solutions which is important in ensuring the optimality of the solution [64]. In the modified genetic algorithm, a random population is initialised, and mutation, crossover and recombination are conducted to generate the offspring population. Sorting is performed to select optimal solutions that are used as parent population for the next iteration. Fig. 3 shows the schematic for encoding in the current implementation for the NSGA-II. Various responses of interest make up the chromosomes, and the process parameters of interest are represented by the gene (represented by $a_{n}$ and $b_{n}$ ), which can be encoded using binaries. 


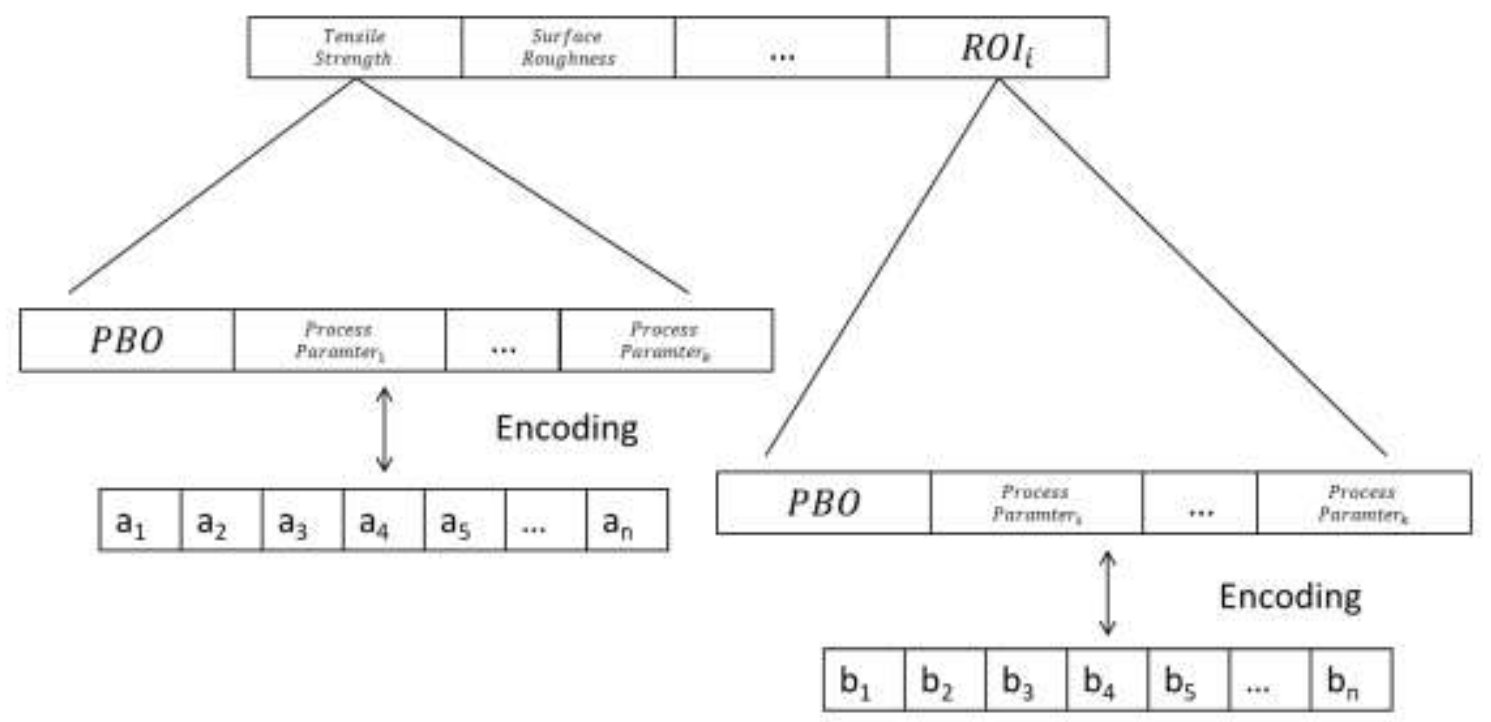

Fig. 3 Chromosome encoding pattern in current implementation of NSGA-II

\subsection{Multiple Criteria Decision-Making Methods}

In MOO, typically, there is no single point for a global optimal solution. Often, a set of solutions is generated which can fulfil the predetermined constrains and meet the definition of optimum. Such set of solution is known as pareto front [60]. From the resultant pareto front, AM designers will select the most optimal solutions for implementation. The solutions should be selected according to how well these design points fit the AM part requirements. Multiple Criteria Decision Making (MCDM) methods are developed to evaluate solutions in situations where there are multiple conflicting criteria. Common methods for MCDM includes Simple Additive Weighting (SAW), Analytic Hierarchy Process (AHP), etc. [65].

In the proposed framework, Technique for Order of Preference by Similarity to Ideal Solution (TOPSIS) is chosen as the MCDM method, due to its universality and ease of implementation. TOPSIS is based on selecting an optimal solution on the shortest Euclidian distance from the Positive Ideal Solution (the Ideal solution) and the farthest from the Negative Ideal Solution (the Nadir solution) [66]. Based on the importance of response, AM designer defines weightage of each response criteria. The TOPSIS algorithm then ranks the solutions from the pareto front, and the optimal solution (i.e. Rank 1) is selected.

\subsection{Empirical validation}

Lastly, the performance of manufactured optimised part can be validated against the generated process maps. Further, data from the validation can be included into the database for future reference and model updating as discussed in Section 2.3. 


\section{Case Study}

In a case study, the proposed framework is illustrated to predict and optimise FFF parts, printed using ULTEM ${ }^{\mathrm{TM}}$ 9085. ULTEM ${ }^{\mathrm{TM}} 9085$ is a thermoplastic co-polymer and utilised in aviation industries due to its ability to retain good mechanical properties at elevated temperature and able to meet flame, smoke, and toxicity requirements [67-71]. However, due to its cost and difficulty in processing, ULTEM ${ }^{\mathrm{TM}}$ 9085 is not widely characterised and studied.

In this case study, a C-shaped clip is selected to demonstrate the applicability of the proposed framework for supporting AM designers in obtaining the optimal design. Fig. 4 shows the CAD model of the clip. Loading is applied to the handle of the part as shown in Fig. 4(a) and the clip requires to have an overall elongation of greater than $1.8 \mathrm{~mm}(\sim 2 \%)$ to ensure the part functionality. To prevent premature failure, Von Mises failure criterion, which has been validated to have good agreement with predicting the failure of FFF parts, is utilised to predict the resultant stress $[72,73]$. The loading is simulated in ABAQUS, as shown in Fig. 4(b). After taking into consideration factor of safety, the required material strength is $60 \mathrm{MPa}$. Further, surface roughness is identified as another part design requirement, as it can influence the durability and fatigue life of AM parts [74-76]. With these part functional requirements defined, the proposed framework can be used to optimise the performance of the FFF part.
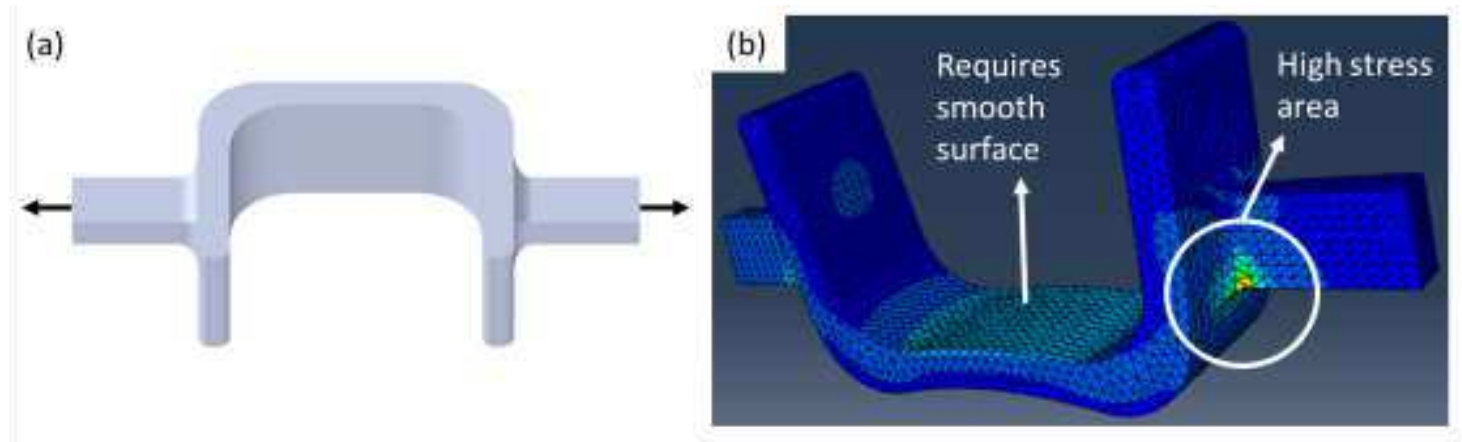

Fig. 4 Part used for verification of framework (a) loading applied onto the part (b) functional requirements defined

\subsection{Sampling of design space}

In this case study, S-optimality LHS is utilised, in which Euclidean distances between each of the design point is maximized, which the enables the correlation between the factors and statistical moments 
to be estimated effectively [77]. To establish the database, factors investigated are PBO and 3 additional process parameters.

PBO is determined to be one of the most critical process parameters influencing a wide variety of response of interest [78-81]. In order to characterize the influence of PBO on response of interest in 3dimensional space, Euler rotation matrix is used, which is described in Equations (7) to (9) below.

$$
\begin{aligned}
& R_{x}=\left[\begin{array}{ccc}
1 & 0 & 0 \\
0 & \cos \alpha & -\sin \alpha \\
0 & \sin \alpha & \cos \alpha
\end{array}\right] \\
& R_{y}=\left[\begin{array}{ccc}
\cos \beta & 0 & \sin \beta \\
0 & 1 & 0 \\
-\sin \beta & 0 & \cos \beta
\end{array}\right]
\end{aligned}
$$

$$
\text { Rotation Matrix, } R=R_{x} R_{y}
$$

The rotation matrix as shown in Equation (9) is used for defining the extrinsic rotation between the local co-ordinate system $(x, y, z)$ with the global co-ordinate system $(X, Y, Z)$ as shown in Fig. 5 (a). Global co-ordinates in AM defines the orientation and direction of a part in the build chamber or on a build platform. As such, building direction of specimens will be in the direction of $+\mathrm{Z}$ axis. Further, right-hand rule defines the positive rotation along an axis.

(a)

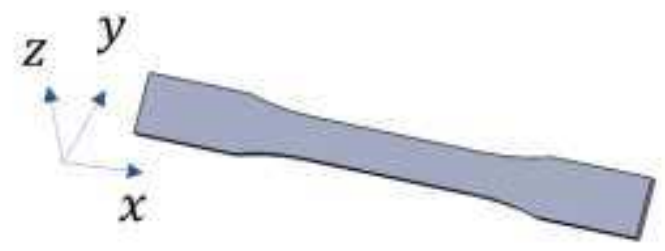

(b) 2

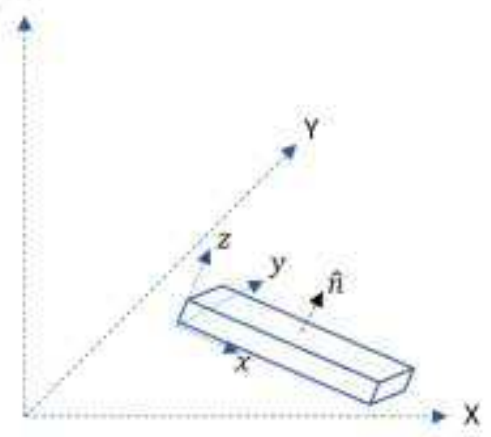

Fig. 5 (a) Standard specimen (ASTM D638 Type I) used for characterization (b) Global co-ordinates and local coordinates

Due to the presence of symmetry of the specimen in the local $z$ axis, the toolpath configuration does not change with $\mathrm{z}$ axis rotation [82]. Thus, only rotations about $x$ and $y$ axes are considered. Equations (10) and (11) show the rotation of the unit vector onto the global co-ordinate system for the loading direction and the evaluation of roughness respectively. 


$$
\begin{gathered}
\text { Surface Roughness }=R^{-1} \cdot\left[\begin{array}{l}
1 \\
1 \\
0
\end{array}\right] \\
\text { Loading direction }=R^{-1} \cdot\left[\begin{array}{l}
0 \\
1 \\
1
\end{array}\right]
\end{gathered}
$$

Surface roughness is defined by the following equation:

$$
R_{a}=\frac{1}{l} \int_{0}^{l}|f(x)| d x
$$

Where $l$ is the length that is evaluated and the $f(x)$ is the surface profile.

Other than PBO, 3 additional process parameters are investigated. These parameters are identified as high impact parameters and are listed and discussed as follow. Refer to Fig. 6 for the associated nomenclature.

- $\quad$ Raster Width (RW): Defines the width of the extruded raster.

- Raster-Raster Air Gap (RRAG): Defines the degree of overlap between the adjacent rasters. Due to ovality of the extruded rasters, gaps are present between adjacent rasters, and a negative airgap closes these gaps. However, if the overlap exceeds a predefined threshold, the extruded raster will cause unevenness on the surface of the print.

- Raster Angle (RA): Defines the angle which the rasters are printed. Angles are defined in the positive X-Y cartesian co-ordinate systems, and the angles are defined with respect to the build chamber. (i.e. rotation of the part does not change the raster angle). The RA of subsequent layer is offset by $90^{\circ}$ to ensure the properties in transverse and longitudinal direction are well balanced.

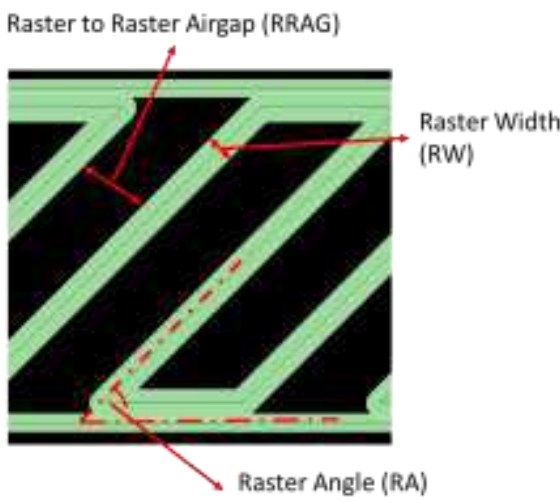

Fig. 6 Nomenclature of process parameters

The selected parameters are commonly investigated in literature as the critical parameters which influences the mechanical response of interest, i.e., mechanical strength and surface roughness [81, 83-86]. Table A1 in Appendix shows the unit S-optimal LHS which is used in this research. Following equations 
(13) -(17) shows the corresponding bounding range and conversion between nominal values and normalised values of the parameters.

$$
\begin{array}{cc}
X=[0,1] * 90 & X:(0,90) \\
Y=[0,1] * 90 & Y=(0,90) \\
R R A G=[0,1] * 0.0254-0.0254 & R R A G:(-0.0254,0) \\
R W=[0,1] *(0.7814-0.4064)+0.4064 & R W:(0.4064,0.7814) \\
R A=[0,1] * 90 & R A:(0,90)
\end{array}
$$

The resultant LHS design matrix is as shown in Fig. 7 as the form of multivariate plot. The plot demonstrates the homogeneity of the design points, which is critical in ensuring the design space to be characterised efficiently. Once the design matrix is defined, the specimens as shown in Fig. 5(a) is printed on Fortus 450MC using ULTEM ${ }^{\mathrm{TM}}$ 9085. There a few main reasons of using ASTM D638 Type 1 for characterisation of both surface roughness and tensile strength: 1) Only one response can be characterised each time, if specimens similar to those utilised in Rahmati and Vahabli [87] were used. As a result, biases may be introduced inadvertently in the data points due to manufacturing variations. 2) Reducing the number of characterisation specimens - such specimens can be time consuming and expensive to manufacture in large quantities 3) ASTM D638 is still one of the most widely used specimens to characterise strength of FFF parts in literature. Hence, to have a basis of comparison, specimens from the standard is utilised.

The surface roughness of the specimen was determined via Keyence Confocal Microscope with laser scanning capabilities for non-contact determination of the surface profile of the specimen according to ISO 25178. On the other hand, tensile test was performed using a Shimadzu 10kN tensile test machine with $0.1 \mathrm{~mm}^{-1}$ crosshead speed, tested according to ASTM 638. Once empirical data is collected, they are then used to train a surrogate model. To increase the number of data points for surface roughness, characterisation is carried out on all sides of the specimen (i.e. specimens are rotated $+90^{\circ},-90^{\circ},+180^{\circ}$ about the $x$ axis). Thus, 3 additional data points for the surface roughness are collected from each of the specimen, which resulted in a total 120 data points. The number of data points for the tensile strength remains at 30 . 


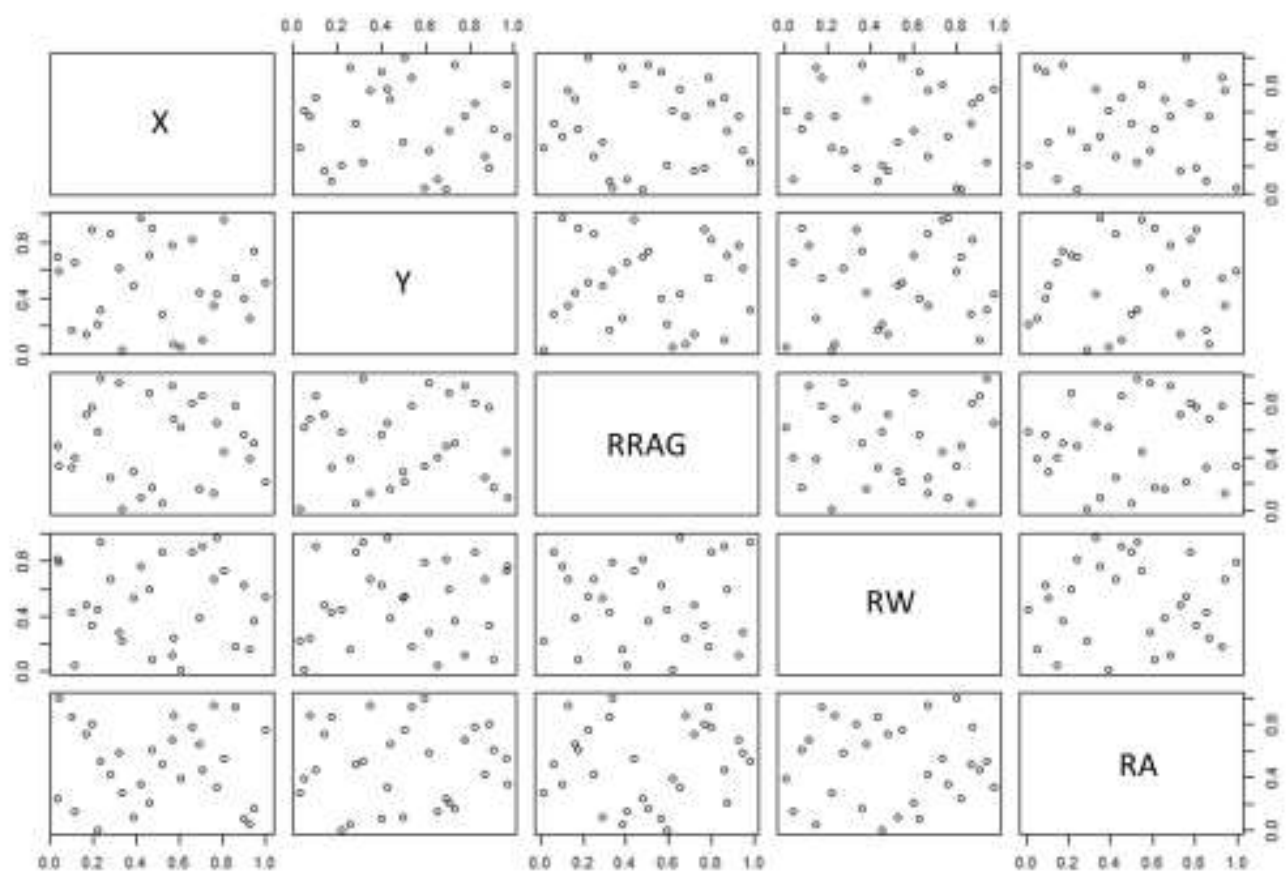

Fig. 7 Multivariate distribution of the LHS for each of the 5 parameters investigated

\subsection{Establishing GPR surrogate model}

In the case study, the covariance function, $K_{\theta}$, is chosen to be the exponential radial basis function $(\mathrm{RBF})$ as shown in Equation (18).

$$
K_{\theta}\left(x, x^{\prime}\right)=e^{-\sum_{k=1}^{p}\left(x_{k}-x_{k}^{\prime}\right) / \theta}
$$

Exponential functions is usually chosen as it is infinitely differentiable, which provides the advantage of being infinitely mean-square differentiable [88]. Comparison against other covariance structure, such as Matérn and linear, will be performed to illustrate the suitability and accuracy of RBF covariance function. The length scale hyperparameter, $\theta$, simulates the exponential decay of the further data points, and the exponential RBF allows for smooth approximation of the modelling over large distances as it is infinitely divisible.

In this case study, anisotropic or separable GPR, where the length scale hyperparameter, $\theta$, differs for each of the variables, is used for modelling of surface roughness and ultimate tensile strength. Weakly informative priors are assigned for hyperparameters $\theta$ using maximum likelihood estimate. Once the GPR 
is trained to predict the mechanical strength and surface roughness, the model is validated for its accuracy using new empirical data before they are used for establishing the process map and the MOO.

\subsection{Validation of surrogate model}

In this section, K-fold cross validation is used to validate the surrogate model's performance, with $K=5$. Root Mean Squared Error, as shown in Equation (19), is used as the comparison metric. Performance of the proposed GPR method is benchmarked against artificial neural network (ANN), support vector regression (SVR) and GPR with other covariance structures, as discussed in previous sections.

$$
R M S E=\frac{1}{n} \sum_{i=1}^{n}\left(\widehat{y}_{i}-y_{i}\right)^{2}
$$

Where $\widehat{y_{l}}$ refers to the predicted $y_{i}$ is the actual value, and $n$ is the number of samples.

Table 1 Accuracy of 5-fold cross validation of models for ultimate tensile strength

\begin{tabular}{|c|c|}
\hline Surrogate modelling technique & RMSE of K-fold cross validation \\
\hline ANN & 9.8 \\
\hline SVR & 10.7 \\
\hline GPR with 'Matérn' covariance & 11.4 \\
\hline GPR with 'linear' covariance & 9.2 \\
\hline GPR with 'RBF' covariance & 9.1 \\
\hline
\end{tabular}

Table 2 Accuracy of 5-fold cross validation of models for surface roughness

\begin{tabular}{|c|c|}
\hline Surrogate modelling technique & RMSE of K-fold cross validation \\
\hline ANN & 13.5 \\
\hline SVR & 13.2 \\
\hline GPR with 'Matern' covariance & 13.1 \\
\hline GPR with 'linear' covariance & 12.9 \\
\hline GPR with 'RBF' covariance & 12.5 \\
\hline
\end{tabular}

As evident from both Tables 1 and 2, performance of GPR with 'RBF' covariance outperforms the rest of the surrogate modelling techniques (lowest RMSE from the K-fold cross validation), especially when compared with more popular techniques ANN and SVR. Further, it is noted that to train an ANN surrogate model, it takes up to 5 to 10 times longer as compared to training a GPR surrogate model of the 
same size. When comparing between the different covariance function used for the GPR method, the accuracy differs lesser, with 'RBF' covariance function is still marginally better as compared to Matérn or Linear covariance function.

\subsection{Process Mapping}

To understand the trade-offs between the various process parameters for FFF, the following process maps are established with respect to the identified process parameters. For the process parameters which are not shown, they were taken at nominal values. Fig. 8 and Fig. 9 present the plots of the relationship between the parameters investigated.

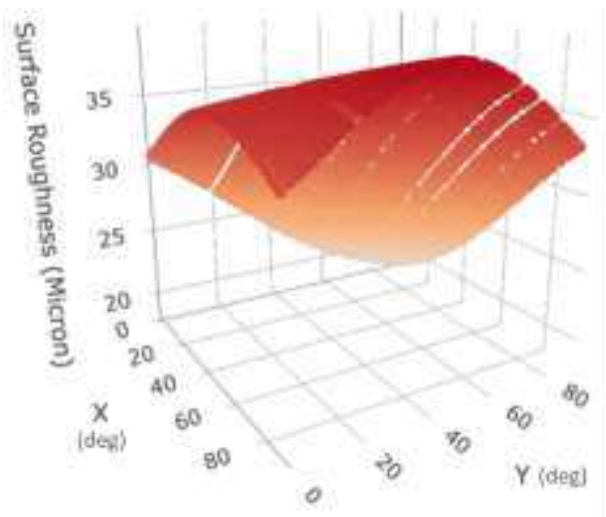

Fig. 8 Variation of surface roughness with rotations about $\mathrm{X} \& \mathrm{Y}$ axis

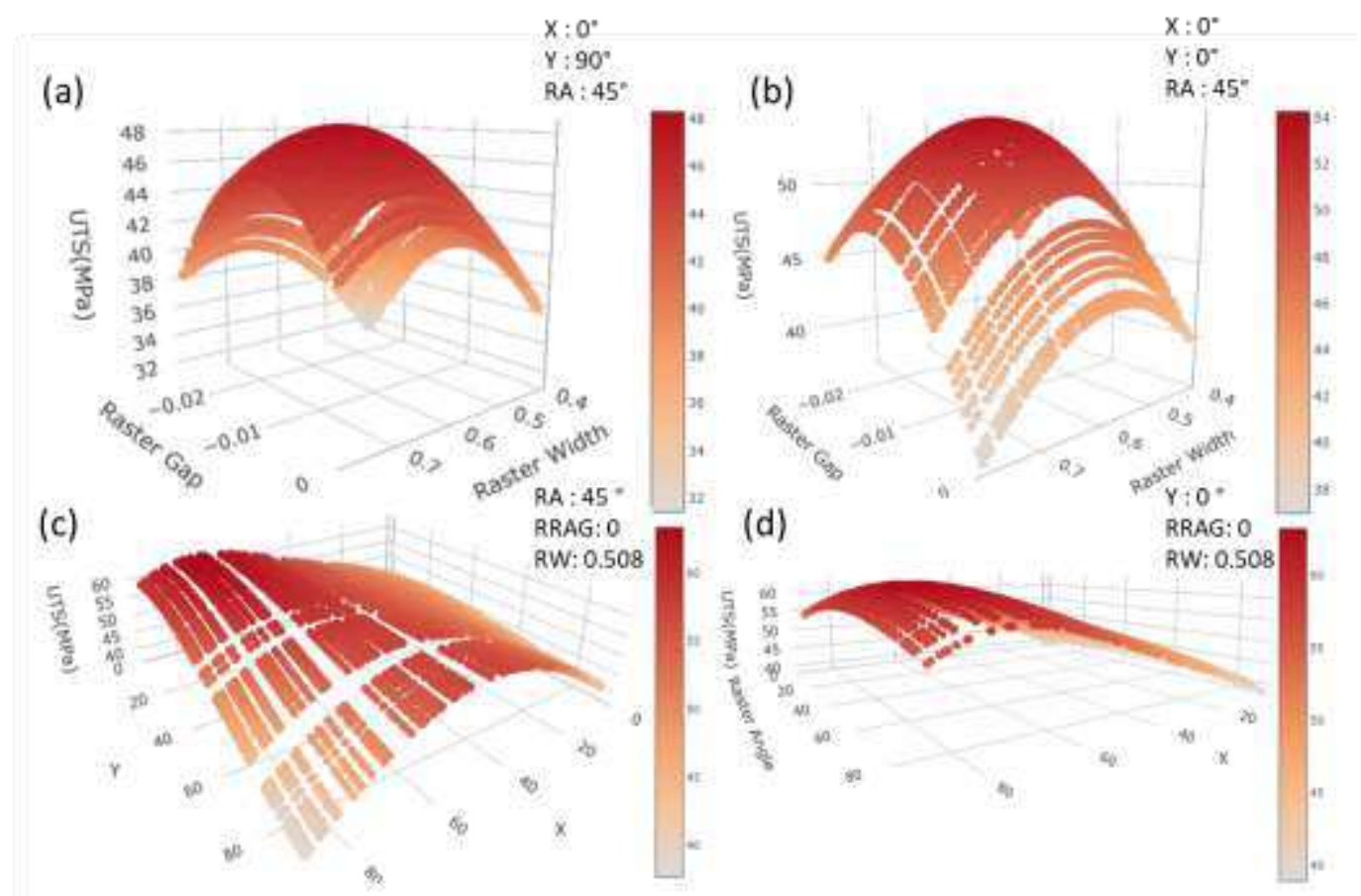

Fig. 9 Variation of UTS with respect to (a) Raster Gap \& Raster Width (with Y rotated 90 deg), (b) Raster Gap \& Raster Width (with Y at 0 deg), (c) Effect of build orientation on mechanical properties (d) Raster Angle \& rotation about $\mathrm{X}$ axis 
Fig. 8 shows the variation of surface roughness with respect to the rotation about the $\mathrm{X} \& \mathrm{Y}$ axes, which reflects the intricate and complex interaction between the response of interest and the rotation of a part in both axes. Most literature only explores the variance of surface roughness along a rotation about a single axis. For example, in Rahmati and Vahabli [87], Vahabli and Rahmati [89] and Boschetto, Giordano [90], the authors explored the relationship between surface roughness and rotation about X axis. Similar trend is observed in mentioned literature can also be seen in Fig. 8. At low angles of rotation about $\mathrm{X}$ axis, the surface roughness is low. As the rotation angle increases, the roughness increases and reaches a maximum of $60^{\circ}$. As the rotation is further increased, the surface roughness decreases.

The variation of ultimate tensile strength with respect to the various process parameters and build orientations are presented in Fig. 9. Both Fig. 9 (a) and (b) show the variations of UTS with respect to raster gap and raster width, except in Fig. 9 (a), the Y axis is rotated 90 deg. The impact of the process parameters is highly dependent on the rotation of the part. When a part is rotated about $\mathrm{Y}$ axis, the impact of changes in RRAG and RW on UTS decreases. Fig. 9 (c) shows the variation of UTS with respect to rotations about $\mathrm{X} \& \mathrm{Y}$ axis. It is apparent that there is confounding between rotations about $\mathrm{X} \& \mathrm{Y}$ axis on the UTS. Simultaneous rotations about X \& Y resulted in a larger drop in UTS. Further, the variation of UTS of rotation about y axis is in good agreement as to what is shown in Zhao, Chen [72]. Similarly, in Fig. 9 (d), the relationship between the raster angle and the rotation about $\mathrm{x}$ axis is determined. From the figure, $45^{\circ}$ RA provides the highest UTS, which is in agreement with observations made by Forés-Garriga, Pérez [86] and Kaplun, Zhou [91].

As compared to state-of-the-art where point wise parameter optimization is performed, process mapping allows AM designers to understand the impact of change of parameters and potential cofounding on the response of interest that may be involved [92, 93]. Further, the knowing how much a response changes by, for a change in process parameter enables for robust design - where parameters can be changed and the variance in the output is minimized.

\subsection{Process Optimisation}

After surrogate models were validated, the MOO was performed. The following Equation (20) shows the objective function for tensile strength (UTS) and surface roughness (SR). Design space, $\Omega$, is as per defined in Equations (13) to (17). A schematic of the MOO process is shown in Fig. 10. Table 3 illustrates the parameters used for NSGA-II. 


$$
\begin{gathered}
\text { Minimise } Y(\boldsymbol{x})=\{-\operatorname{UTS}(\boldsymbol{x}), S R(\boldsymbol{x})\}^{T} \\
\text { where } \boldsymbol{x}=X, Y, R R A G, R W, R A \\
\text { s.t.UTS }(\boldsymbol{x})-60 \geq 0 \\
\boldsymbol{x} \in \Omega
\end{gathered}
$$

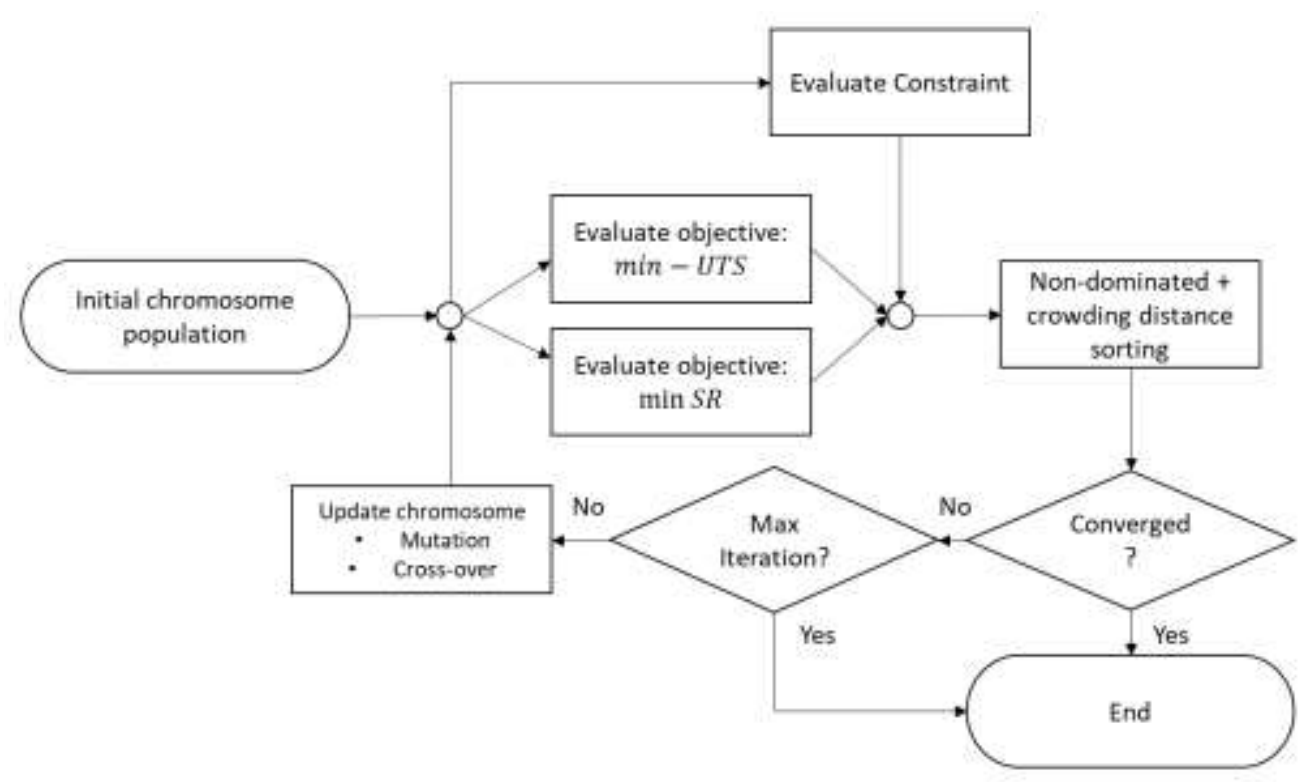

Fig. 10 MOO implementation for the case study

Table 3 Parameters used in NSGA-II

\begin{tabular}{|c|c|}
\hline Parameters & Settings \\
\hline Population size & 100 \\
\hline Generations & 1000 \\
\hline Crossover Probability & 0.7 \\
\hline Mutation Probability & 0.2 \\
\hline
\end{tabular}

Pareto front from the MOO is generated as shown in Fig. 11(a), where each solution is represented by a point. As evident from the graph, the trend is monotonically increases (i.e. as tensile strength increases, surface roughness increase), which provides proof that these 2 responses are in fact conflicting. One the pareto front is established, solutions were checked that it falls within the feasible space (any infeasible ones were removed), and MCDM was performed on the solution set using TOPSIS. Equal weights were assigned to both tensile strength and surface roughness. The solutions were ranked according to their preference score, and 3 solutions of different ranks (top, middle and bottom) where chosen from the pareto front for validation, to check if the results were consistent against prediction across the whole solution set. The 
solutions with their associated parameter set are presented in Table 4. The clips are then orientated in the Insight slicer and the parameters assigned to the prints are shown in Table 4. The clips are printed in the same build, and once the build is completed, the support is removed, and they are sent for testing. Fig. 11(b) shows the test setup for part validate the results and accuracy of the prediction model.

(a)

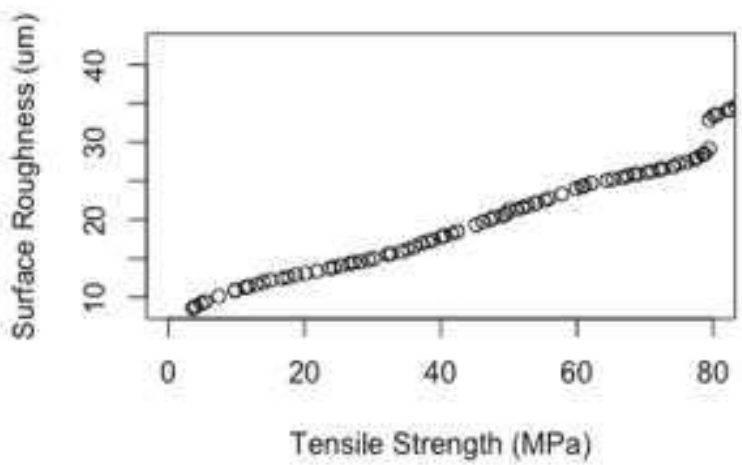

(b)

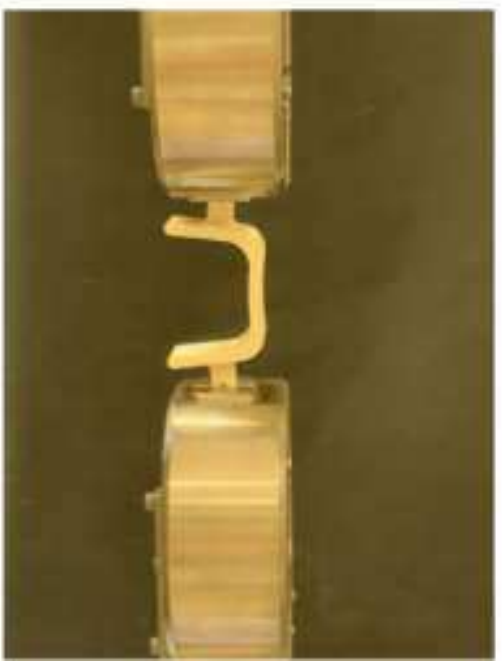

Fig. 11 (a) Pareto front for MOO (b) Part testing of resultant solutions

Table 4 Ranked pareto front according to TOPSIS

\begin{tabular}{|c|c|c|c|c|c|c|c|c|}
\hline Rank & $\begin{array}{l}>\text { Required } \\
\text { strength }\end{array}$ & $\begin{array}{c}\text { SR } \\
(\boldsymbol{\mu m})\end{array}$ & $\mathbf{X}\left(^{\circ}\right)$ & $\mathbf{Y}\left(^{\circ}\right)$ & $\begin{array}{c}\text { Raster- } \\
\text { RasterA } \\
\mathbf{i r ~ G a p} \\
(\boldsymbol{\mu m})\end{array}$ & $\begin{array}{c}\text { Raster } \\
\text { Width } \\
(\boldsymbol{\mu m})\end{array}$ & $\begin{array}{c}\text { Raste } \\
\mathbf{r} \\
\text { Angle } \\
\left({ }^{\circ}\right)\end{array}$ & TOPSIS \\
\hline 1 & Yes & 26.7 & 34.6 & 90.0 & -0.01 & 0.58 & 0.27 & 0.82 \\
\hline 8 & Yes & 31.5 & 47.0 & 68.5 & -0.02 & 0.57 & 0.90 & 0.72 \\
\hline 39 & Yes & 49.4 & 77.7 & 5.8 & -0.02 & 0.60 & 1.53 & 0.19 \\
\hline
\end{tabular}

Table 5 Actual and predicted results for surface roughness of defined location

\begin{tabular}{|c|c|c|c|}
\hline Rank & Predicted SR $(\boldsymbol{\mu m})$ & Actual SR $(\boldsymbol{\mu m})$ & SR RE $(\boldsymbol{\%})$ \\
\hline 1 & 26.67 & 28.16 & 5.3 \\
\hline 8 & 31.53 & 29.96 & 5.2 \\
\hline 39 & 49.45 & 64.85 & 24 \\
\hline
\end{tabular}

All the 3 printed parts were able to pass the elongation requirement as shown in Table 5 , showing the results for the measured surface roughness at the location as defined in Fig. 4. For both Rank 1 and Rank 8 solutions, the prediction results are relatively close to the actual results, with a relative error of approximately 5\%. However, for Rank 39 solution, the prediction under predicts the actual surface 
roughness by a relatively wide margin $(24 \%)$. It is postulated that more datapoints can further enhance the predictive accuracy in terms of surface roughness.

\subsection{Discussion}

Through the case study of designing a C-shaped clip using FFF ULTEM ${ }^{\mathrm{TM}}$ 9085, the efficacy of the proposed framework is proven, which enabled an AM part to be designed to meet the part functional requirements. With relatively small dataset, the validation results show that the prediction agrees well with empirical data. Further, FFF process maps generated using the validated surrogate model enable to gain valuable insights about the influence of process parameters PBO and FFF on response of interest, such as tensile strength and part surface roughness. The process maps, generated from this single characterisation, can present important trends and relationships between process parameters that often only apparent across multiple investigation studies in literatures. As evident, adopting a data-driven approach enables large design spaces to be characterised efficiently and effectively, and enabling optimal part performance to be achieved with relative ease.

\section{Conclusions and Future Work}

In this research, a data driven framework was proposed to achieve the optimal AM part performance through prediction and part optimisation. GPR surrogate modelling and optimised Latin Hypercube Sampling were utilised for efficient characterisation of the enlarged design space. Process maps generated from these established and validated surrogate models were then used for understanding the nuances in AM process. Optimisation based on validated surrogate models was carried out and MCDM is utilised to pinpoint the optimal design point. The proposed framework was then validated through a case study by predicting and optimising a FFF part performance to meet the design requirements. Through the case study, important insights from FFF process can be established, and trends and relationships between critical parameters can be visually represented through process maps. The process maps, generated from this single characterisation, can present important trends and relationships between process parameters that often only apparent across multiple investigation studies in literatures. This highlights the efficiency of the proposed framework. Once the surrogate models are validated, prediction and optimisation of response of interests can be performed using MOO methods. MCDM methods can then be utilised to identify the optimal design point. This enables AM designers to achieve optimal design for FFF parts, even within large design space dimensions due to PSP linkages, efficiently and effectively. 
Future work includes further improving the prediction accuracy incorporating data from various sources, such as from available literature, and performing experiment on design points which provide the greatest expected information. Moreover, increasing the dimensions of design space, such as simultaneous optimisation and trade-offs between many more AM responses of interest and other process parameters can be performed, which can further accentuate the advantage of adopting the proposed framework in designing AM parts. Lastly, the proposed framework can be further extended and validated using other AM technologies. 


\section{References}

1. Jiang, J. and Y. Ma, Path planning strategies to optimize accuracy, quality, build time and material use in additive manufacturing: a review. Micromachines, 2020. 11(7): p. 633.

2. Jiang, J., A novel fabrication strategy for additive manufacturing processes. Journal of Cleaner Production, 2020. 272: p. 122916.

3. Hu, F. and D. Wu. Cellular Structures Design for Wrist Rehabilitation Considering 3D Printability and Mechanics Lightweight. in 2019 WRC Symposium on Advanced Robotics and Automation (WRC SARA). 2019. Beijing, China: IEEE.

4. Wu, D., Y. Wei, and J. Terpenny, Surface Roughness Prediction in Additive Manufacturing Using Machine Learning, in ASME 2018 13th International Manufacturing Science and Engineering Conference. 2018, American Society of Mechanical Engineers Digital Collection: College Station, Texas, USA.

5. Mineo, C., et al., Introducing a novel mesh following technique for approximation-free robotic tool path trajectories. Journal of Computational Design and Engineering, 2017. 4(3): p. 192-202.

6. Kim, J. and D.-J. Yoo, 3D printed compact heat exchangers with mathematically defined core structures. Journal of Computational Design and Engineering, 2020. 7(4): p. 527-550.

7. Goh, G., et al., Additively manufactured continuous carbon fiber reinforced thermoplastic for topology optimized unmanned aerial vehicle structures. Composites Part B: Engineering, 2021. 216: p. 108840.

8. Vantyghem, G., et al., 3D printing of a post-tensioned concrete girder designed by topology optimization. Automation in Construction, 2020. 112: p. 103084.

9. Khodaygan, S. and A. Golmohammadi, Multi-criteria optimization of the part build orientation (PBO) through a combined meta-modeling/NSGAII/TOPSIS method for additive manufacturing processes. International Journal on Interactive Design and Manufacturing (IJIDeM), 2018. 12(3): p. 1071-1085.

10. Golmohammadi, A. and S. Khodaygan, A framework for multi-objective optimisation of $3 D$ partbuild orientation with a desired angular resolution in additive manufacturing processes. Virtual and Physical Prototyping, 2019. 14(1): p. 19-36.

11. Pandey, P.M., K. Thrimurthulu, and N.V. Reddy, Optimal part deposition orientation in FDM by using a multicriteria genetic algorithm. International Journal of Production Research, 2004. 42(19): p. 4069-4089.

12. Zhang, Y. and S.K. Moon, Data-driven design strategy in fused filament fabrication: status and opportunities. Journal of Computational Design and Engineering, 2021.

13. Zhang, H., et al., Rapid Process Modeling of the Aerosol Jet Printing Based on Gaussian Process Regression with Latin Hypercube Sampling. International Journal of Precision Engineering and Manufacturing, 2019(21): p. 127-136.

14. Rankouhi, B., et al., Compositional Grading of a 316L-Cu Multi-material Part Using Machine Learning for the Determination of Selective Laser Melting Process Parameters. Additive Manufacturing, 2021: p. 101836.

15. An, D., N.H. Kim, and J.-H. Choi, Practical options for selecting data-driven or physics-based prognostics algorithms with reviews. Reliability Engineering \& System Safety, 2015. 133: p. 223236.

16. Qi, X., et al., Applying neural-network-based machine learning to additive manufacturing: current applications, challenges, and future perspectives. Engineering, 2019. 5(4): p. 721-729.

17. Goh, G.D., S.L. Sing, and W.Y. Yeong, A review on machine learning in 3D printing: applications, potential, and challenges. Artificial Intelligence Review, 2020: p. 1-32.

18. Mycroft, W., et al., A data-driven approach for predicting printability in metal additive manufacturing processes. Journal of Intelligent Manufacturing, 2020. 31: p. 1769-1781.

19. Aminzadeh, M. and T.R. Kurfess, Online quality inspection using Bayesian classification in powderbed additive manufacturing from high-resolution visual camera images. Journal of Intelligent Manufacturing, 2019. 30(6): p. 2505-2523.

20. Xiong, Y., et al., Data-driven design space exploration and exploitation for design for additive manufacturing. Journal of Mechanical Design, 2019. 141(10): p. 101101.

21. Jiang, J., et al., Machine learning integrated design for additive manufacturing. Journal of Intelligent Manufacturing, 2020: p. 1-14.

22. Paul, A., et al., A real-time iterative machine learning approach for temperature profile prediction in additive manufacturing processes. arXiv preprint arXiv:1907.12953, 2019. 
23. Roy, M. and O. Wodo, Data-driven modeling of thermal history in additive manufacturing. Additive Manufacturing, 2020. 32: p. 101017.

24. Yang, X., et al., Towards a process-structure model for Ti-6Al-4V during additive manufacturing. Journal of Manufacturing Processes, 2021. 61: p. 428-439.

25. Jiang, J., et al., Analysis and prediction of printable bridge length in fused deposition modelling based on back propagation neural network. Virtual and Physical Prototyping, 2019. 14(3): p. 253266.

26. Jothibabu, G. and S. Kumar, Surrogate Based Sensitivity Analysis of Part Strength due to Process Parameters in Fused Deposition Modelling. Proced. Comput. Sci, 2018. 133: p. 772-778.

27. Vahabli, E. and S. Rahmati, Application of an RBF neural network for FDM parts'surface roughness prediction for enhancing surface quality. International journal of precision engineering and manufacturing, 2016. 17(12): p. 1589-1603.

28. Liu, Y. and P. Wang. Probabilistic modeling and analysis of fused deposition modeling process using surrogate models. in ASME 2016 International Design Engineering Technical Conferences and Computers and Information in Engineering Conference. 2016. American Society of Mechanical Engineers Digital Collection.

29. Standard, A., ISO/ASTM 52900: 2015 Additive manufacturing-General principles-terminology. ASTM F2792-10e1, 2012.

30. Chua, C.K. and K.F. Leong, 3D Printing and Additive Manufacturing: Principles and Applications Fifth Edition of Rapid Prototyping 5th Edition. 2017, Singapore: World Scientifc.

31. Sathies, T., P. Senthil, and M. Anoop, A review on advancements in applications of fused deposition modelling process. Rapid Prototyping Journal, 2020. 26(4): p. 669-687.

32. Vyavahare, S., et al., Fused deposition modelling: A review. Rapid Prototyping Journal, 2020. 26(1): p. 176-201.

33. George N. Szatkowski, L.A.T., Evaluation of Ultem 1000, 1010 and 9085 for Radome Applications at 24.5 GHz. 2019, National Aeronautics and Space Administration.

34. Hardin, W. Additive Manufacturing Boosts Aerospace Applications. 2016 [cited 202116 October]; Available from: https://insights.globalspec.com/article/1885/additive-manufacturing-boostsaerospace-applications.

35. Pearson, A. Stratasys 3D printing keeps NASA satellite on time and on budget. 2020 [cited 202116 October]; Available from: https://www.stratasys.com/explore/blog/2015/nasa-3d-printing-fortus.

36. Stratasys. Stratasys Direct Manufacturing Builds the First 3D Printed Parts to Function on the Exterior of a Satellite. Available from: https://www.stratasysdirect.com/resources/case-studies/3dprinted-satellite-exterior-nasa-jet-propulsion-laboratory.

37. Grady, J.E., A Fully Non-Metallic Gas Turbine Engine Enabled by Additive Manufacturing. 2014, NASA Gleen Research Centre.

38. Tapia, G., A. Elwany, and H. Sang, Prediction of porosity in metal-based additive manufacturing using spatial Gaussian process models. Additive Manufacturing, 2016. 12: p. 282-290.

39. Seifi, M., et al. Process mapping, fracture and fatigue behavior of Ti-6Al-4V produced by EBM additive manufacturing. in Proceedings of the 13th World Conference on Titanium. 2016. Citeseer.

40. Montgomery, C., et al. Process mapping of Inconel 625 in laser powder bed additive manufacturing. in Solid freeform fabrication symposium. 2015.

41. Zhu, J.-N., et al., Predictive analytical modelling and experimental validation of processing maps in additive manufacturing of nitinol alloys. Additive Manufacturing, 2021. 38: p. 101802.

42. Fang, K.-T., et al., Uniform design: theory and application. Technometrics, 2000. 42(3): p. 237-248.

43. Zhang, H., et al., A multi-objective optimization framework for aerosol jet customized line width printing via small data set and prediction uncertainty. Journal of Materials Processing Technology, 2020: p. 116779.

44. Wang, Z., et al., A Data-Driven Approach for Process Optimization of Metallic Additive Manufacturing Under Uncertainty. Journal of Manufacturing Science and Engineering, 2019. 141(8): p. 081004-1.

45. Tapia, G., et al., Gaussian process-based surrogate modeling framework for process planning in laser powder-bed fusion additive manufacturing of $316 \mathrm{~L}$ stainless steel. The International Journal of Advanced Manufacturing Technology, 2018. 94(9-12): p. 3591-3603.

46. Congdon, P.D., Bayesian Hierarchical Models: With Applications Using R. 2019: CRC Press.

47. Chong, A. and K. Menberg, Guidelines for the Bayesian calibration of building energy models. Energy and Buildings, 2018. 174: p. 527-547.

48. Gramacy, R.B., Surrogates: Gaussian Process Modeling, Design, and Optimization for the Applied 
Sciences. 2020: CRC Press.

49. Tapia, G., et al., Bayesian calibration and uncertainty quantification for a physics-based precipitation model of nickel-titanium shape-memory alloys. Journal of Manufacturing Science and Engineering, 2017. 139(7).

50. Gramacy, R.B., laGP: large-scale spatial modeling via local approximate Gaussian processes in $R$. Journal of Statistical Software, 2016. 72(1): p. 1-46.

51. Simpson, T., et al. Comparison of response surface and kriging models for multidisciplinary design optimization. in 7th AIAA/USAF/NASA/ISSMO Symposium on Multidisciplinary Analysis and Optimization. 1998.

52. Anguita, D., et al. The 'K' in K-fold cross validation. in 20th European Symposium on Artificial Neural Networks, Computational Intelligence and Machine Learning (ESANN). 2012. i6doc. com publ.

53. Garbo, A. and B.J. German, Performance assessment of a cross-validation sampling strategy with active surrogate model selection. Structural and Multidisciplinary Optimization, 2019. 59(6): p. 2257-2272.

54. Mack, Y., et al., Surrogate model-based optimization framework: a case study in aerospace design, in Evolutionary computation in dynamic and uncertain environments. 2007, Springer. p. 323-342.

55. Wong, T.-T., Performance evaluation of classification algorithms by $k$-fold and leave-one-out cross validation. Pattern Recognition, 2015. 48(9): p. 2839-2846.

56. Jones, D.R., M. Schonlau, and W.J. Welch, Efficient global optimization of expensive black-box functions. Journal of Global optimization, 1998. 13(4): p. 455-492.

57. Aoyagi, K., et al., Simple method to construct process maps for additive manufacturing using a support vector machine. Additive Manufacturing, 2019. 27: p. 353-362.

58. Zhou, A., et al., Multiobjective evolutionary algorithms: A survey of the state of the art. Swarm and Evolutionary Computation, 2011. 1(1): p. 32-49.

59. Fonseca, C.M. and P.J. Fleming, An overview of evolutionary algorithms in multiobjective optimization. Evolutionary computation, 1995. 3(1): p. 1-16.

60. Marler, R.T. and J.S. Arora, Survey of multi-objective optimization methods for engineering. Structural and multidisciplinary optimization, 2004. 26(6): p. 369-395.

61. Deb, K., et al., A fast and elitist multiobjective genetic algorithm: NSGA-II. IEEE transactions on evolutionary computation, 2002. 6(2): p. 182-197.

62. Konak, A., D.W. Coit, and A.E. Smith, Multi-objective optimization using genetic algorithms: A tutorial. Reliability engineering \& system safety, 2006. 91(9): p. 992-1007.

63. Alizadeh, M., et al., Data-Driven Energy Efficiency and Part Geometric Accuracy Modeling and Optimization of Green Fused Filament Fabrication Processes. Journal of Mechanical Design, 2020. 142(4): p. 041701.

64. Coello, C.A.C., Evolutionary multi-objective optimization: A critical review, in Evolutionary optimization. 2003, Springer: Boston, MA. p. 117-146.

65. Mardani, A., et al., Multiple criteria decision-making techniques and their applications-a review of the literature from 2000 to 2014. Economic Research-Ekonomska Istraživanja, 2015. 28(1): p. 516571.

66. Hwang, C.-L. and K. Yoon, Methods for multiple attribute decision making, in Multiple attribute decision making. 1981, Springer. p. 58-191.

67. Stratasys, ULTEM $^{\mathrm{TM}} \quad 9085 . \quad 2016, \quad$ Stratasys: http://usglobalimages.stratasys.com/Main/Files/Material_Spec_Sheets/MSS_FDM_ULTEM9085.p df.

68. Cicala, G., et al., Comparison of Ultem 9085 used in fused deposition modelling (FDM) with polytherimide blends. Materials, 2018. 11(2): p. 285.

69. Blanco, I., et al., Thermal properties of polyetherimide/polycarbonate blends for advanced applications. Polymer Degradation and Stability, 2018. 154: p. 234-238.

70. Ceruti, A., et al., Maintenance in aeronautics in an Industry 4.0 context: The role of Augmented Reality and Additive Manufacturing. Journal of Computational Design and Engineering, 2019. 6(4): p. 516-526.

71. de Bruijn, A.C., G. Gómez-Gras, and M.A. Pérez, Mechanical study on the impact of an effective solvent support-removal methodology for FDM Ultem 9085 parts. Polymer Testing, 2020. 85: p. 106433.

72. Zhao, Y., Y. Chen, and Y. Zhou, Novel mechanical models of tensile strength and elastic property of FDM AM PLA materials: Experimental and theoretical analyses. Materials \& Design, 2019. 181: p. 
108089.

73. Alaimo, G., et al., Influence of meso-structure and chemical composition on FDM 3D-printed parts. Composites Part B: Engineering, 2017. 113: p. 371-380.

74. Fischer, M. and V. Schöppner, Fatigue behavior of FDM parts manufactured with Ultem 9085. Jom, 2017. 69(3): p. 563-568.

75. Gockel, J., et al., The influence of additive manufacturing processing parameters on surface roughness and fatigue life. International Journal of Fatigue, 2019. 124: p. 380-388.

76. Pegues, J., et al., Surface roughness effects on the fatigue strength of additively manufactured Ti-6Al$4 V$. International Journal of Fatigue, 2018. 116: p. 543-552.

77. Stocki, R., A method to improve design reliability using optimal Latin hypercube sampling. Computer Assisted Mechanics and Engineering Sciences, 2005. 12(4): p. 393.

78. Motaparti, K.P., et al., Experimental investigation of effects of build parameters on flexural properties in fused deposition modelling parts. Virtual and Physical Prototyping, 2017. 12(3): p. 207220.

79. Laureto, J.J. and J.M. Pearce, Anisotropic mechanical property variance between ASTM D638-14 type $i$ and type iv fused filament fabricated specimens. Polymer Testing, 2018. 68: p. 294-301.

80. Ding, S., et al., Effects of nozzle temperature and building orientation on mechanical properties and microstructure of PEEK and PEI printed by 3D-FDM. Polymer Testing, 2019. 78: p. 105948.

81. Ahn, D., et al., Representation of surface roughness in fused deposition modeling. Journal of Materials Processing Technology, 2009. 209(15-16): p. 5593-5600.

82. Forés-Garriga, A., et al., Role of infill parameters on the mechanical performance and weight reduction of PEI Ultem processed by FFF. Materials \& Design, 2020: p. 108810.

83. Sood, A.K., R.K. Ohdar, and S.S. Mahapatra, Parametric appraisal of mechanical property of fused deposition modelling processed parts. Materials \& Design, 2010. 31(1): p. 287-295.

84. Gebisa, A.W. and H.G. Lemu, Investigating Effects of Fused-Deposition Modeling (FDM) Processing Parameters on Flexural Properties of ULTEM 9085 using Designed Experiment. Materials, 2018. 11(4): p. 500.

85. Liu, X., et al., Mechanical property parametric appraisal of fused deposition modeling parts based on the gray Taguchi method. The International Journal of Advanced Manufacturing Technology, 2017. 89(5-8): p. 2387-2397.

86. Forés-Garriga, A., et al., Role of infill parameters on the mechanical performance and weight reduction of PEI Ultem processed by FFF. Materials \& Design, 2020. 193: p. 108810.

87. Rahmati, S. and E. Vahabli, Evaluation of analytical modeling for improvement of surface roughness of FDM test part using measurement results. The International Journal of Advanced Manufacturing Technology, 2015. 79(5-8): p. 823-829.

88. Rasmussen, C.E. Gaussian processes in machine learning. in Summer School on Machine Learning. 2003. Springer.

89. Vahabli, E. and S. Rahmati, Hybrid estimation of surface roughness distribution in FDM parts using analytical modeling and empirical investigation. The International Journal of Advanced Manufacturing Technology, 2017. 88(5-8): p. 2287-2303.

90. Boschetto, A., V. Giordano, and F. Veniali, Surface roughness prediction in fused deposition modelling by neural networks. The International Journal of Advanced Manufacturing Technology, 2013. 67(9-12): p. 2727-2742.

91. Kaplun, B.W., et al., Influence of orientation on mechanical properties for high-performance fused filament fabricated ultem 9085 and electro-statically dissipative polyetherketoneketone. Additive Manufacturing, 2020. 36: p. 101527.

92. Mahmood, S., et al., Tensile strength of partially filled FFF printed parts: experimental results. Rapid Prototyping Journal, 2017. 23(1): p. 122-128.

93. Wang, S., et al., Effects of fused deposition modeling process parameters on tensile, dynamic mechanical properties of 3D printed polylactic acid materials. Polymer Testing, 2020. 86: p. 106483. 


\section{Statement \& Declarations}

\section{Funding}

This research is supported by a grant from ST Engineering Aerospace, EDB-IPP, the National Research Foundation, Prime Minister's Office, Singapore under its Medium-Sized Centre funding scheme, and Singapore Centre for 3D Printing.

\section{Competing Interests}

The authors have no competing interests to declare that are relevant to the content of this article.

\section{Author Contributions}

All authors contributed to the study conception and design. Material preparation, data collection and analysis were performed by Yongjie Zhang. The first draft of the manuscript was written by Yongjie Zhang, and all authors reviewed previous versions of the manuscript. All authors read and approved the final manuscript. 


\section{Appendix}

Table A1 Normalized values for X,Y,Z, RRAG, RW, RA

\begin{tabular}{|c|c|c|c|c|c|}
\hline $\mathbf{S} / \mathbf{N}$ & $\mathbf{X}$ & $\mathbf{Y}$ & RRAG & RW & RA \\
\hline 1. & 0.471 & 0.905 & 0.174 & 0.084 & 0.605 \\
\hline 2. & 0.109 & 0.653 & 0.401 & 0.041 & 0.144 \\
\hline 3. & 0.775 & 0.426 & 0.656 & 0.969 & 0.330 \\
\hline 4. & 0.099 & 0.170 & 0.321 & 0.431 & 0.851 \\
\hline 5. & 0.164 & 0.139 & 0.717 & 0.478 & 0.727 \\
\hline 6. & 0.460 & 0.706 & 0.878 & 0.600 & 0.214 \\
\hline 7. & 0.033 & 0.692 & 0.480 & 0.820 & 0.243 \\
\hline 8. & 0.897 & 0.397 & 0.566 & 0.626 & 0.090 \\
\hline 9. & 0.757 & 0.345 & 0.126 & 0.668 & 0.939 \\
\hline 10. & 0.858 & 0.539 & 0.785 & 0.174 & 0.931 \\
\hline 11. & 0.234 & 0.315 & 0.986 & 0.938 & 0.525 \\
\hline 12. & 0.383 & 0.495 & 0.291 & 0.528 & 0.104 \\
\hline 13. & 0.420 & 0.973 & 0.098 & 0.760 & 0.351 \\
\hline 14. & 0.280 & 0.864 & 0.251 & 0.666 & 0.425 \\
\hline 15. & 0.608 & 0.047 & 0.618 & 0.007 & 0.387 \\
\hline 16. & 0.707 & 0.101 & 0.858 & 0.908 & 0.453 \\
\hline 17. & 0.319 & 0.611 & 0.947 & 0.277 & 0.587 \\
\hline 18. & 1.000 & 0.507 & 0.221 & 0.547 & 0.757 \\
\hline 19. & 0.568 & 0.073 & 0.682 & 0.237 & 0.869 \\
\hline 20. & 0.946 & 0.734 & 0.505 & 0.359 & 0.171 \\
\hline 21. & 0.041 & 0.593 & 0.334 & 0.798 & 0.994 \\
\hline 22. & 0.521 & 0.283 & 0.059 & 0.864 & 0.498 \\
\hline 23. & 0.693 & 0.440 & 0.161 & 0.384 & 0.654 \\
\hline
\end{tabular}




\begin{tabular}{|c|c|c|c|c|c|}
\hline 24. & 0.661 & 0.823 & 0.802 & 0.869 & 0.775 \\
\hline 25. & 0.926 & 0.255 & 0.383 & 0.150 & 0.052 \\
\hline 26. & 0.802 & 0.962 & 0.436 & 0.731 & 0.545 \\
\hline 27. & 0.215 & 0.215 & 0.593 & 0.452 & 0.007 \\
\hline 28. & 0.566 & 0.774 & 0.930 & 0.117 & 0.683 \\
\hline 29. & 0.191 & 0.890 & 0.766 & 0.331 & 0.803 \\
\hline 30. & 0.334 & 0.033 & 0.010 & 0.220 & 0.289 \\
\hline
\end{tabular}

